\title{
Static deformation due to a long buried dip-slip fault in an isotropic half-space welded with an orthotropic half-space
}

\author{
NEERU BALA and SUNITA RANI* \\ Department of Mathematics, Guru Jambheshwar University of Science and \\ Technology, Hisar 125001 \\ e-mail: s_b_rani@rediffmail.com
}

MS received 29 April 2008; revised 20 October 2008

\begin{abstract}
Closed-form analytical expressions for the displacements and the stresses at any point of a two-phase medium consisting of a homogeneous, isotropic, perfectly elastic half-space in welded contact with a homogeneous, orthotropic, perfectly elastic half-space due to a dip-slip fault of finite width located at an arbitrary distance from the interface in the isotropic half-space are obtained. The Airy stress function approach is used to obtain the expressions for the stresses and the displacements. The case of a vertical dip-slip fault is considered in detail. The variations of the displacements with the distance from the fault and with depth have been shown graphically.
\end{abstract}

Keywords. Isotropic half-space; dip-slip fault; orthotropic half-space; static deformation; welded contact.

\section{Introduction}

The two half-space model is suitable for computing the deformation caused by seismic sources near an internal boundary. Several researchers have studied the deformation due to shear or tensile faults in the two half-space model e.g. Ben-Zion (1989), Singh \& Rani (1991), Singh et al (1992), Rani \& Singh (1992), Bonafede \& Rivalta (1999), Kumar et al (2002), Kumar et al (2005), Rani \& Bala (2006) and the others. Wu \& Chou (1982) applied the generalized method of images to obtain the elastic field of an in-plane line force acting in a two-phase orthotropic medium. Garg et al (1996) studied the antiplane strain deformation of a layered orthotropic medium. Garg et al (1991) studied the plane strain deformation of an orthotropic elastic half-space using transfer matrix approach.

In the present paper, we study the deformation caused by a long dip-slip fault of arbitrary dip and finite width located at an arbitrary distance from the interface in an isotropic halfspace welded with an orthotropic half-space along the horizontal plane. We begin with the closed-form expression for the Airy stress function due to a dip slip line source given by Singh \& Rani (1991) in a two-phase medium consisting of a homogeneous, elastic, isotropic

${ }^{*}$ For correspondence 
half-space in welded contact with a homogeneous, elastic, orthotropic half-space. Following Rani \& Bala (2006), we obtain the Airy stress function for a long buried dip-slip fault of finite width. The expressions for the displacements and the stresses follow immediately from the Airy stress function. The displacement field due to buried and interface-breaking vertical dip-slip fault has been computed numerically and is compared with the displacement field when both the half-spaces are isotropic. Orthotropic or orthorhombic symmetry is exhibited by olivine and orthopyroxenes, the principal rock forming minerals of deep crust and upper mantle. Therefore, we take the lower half-space to be orthotropic.

\section{Theory}

Let the Cartesian co-ordinates be denoted by $\left(x_{1}, x_{2}, x_{3}\right)$ with $x_{3}$-axis vertically upwards. Consider two homogeneous, perfectly elastic half-spaces which are welded along the plane $x_{3}=0$. The upper half-space $\left(x_{3}>0\right)$ is assumed to be isotropic with stress-strain relation

$$
p_{i j}=2 \mu\left[e_{i j}+\frac{\sigma}{1-2 \sigma} \delta_{i j} e_{k k}\right], \quad(i, j=1,2,3)
$$

where $p_{i j}$ are the components of stress tensor, $e_{i j}$ are the components of strain tensor, $\mu$ is the shear modulus and $\sigma$ is Poisson's ratio. The lower half-space $\left(x_{3}<0\right)$ is assumed to be orthotropic with stress-strain relation

$$
\left[\begin{array}{c}
p_{11}^{\prime} \\
p_{22}^{\prime} \\
p_{33}^{\prime} \\
p_{23}^{\prime} \\
p_{31}^{\prime} \\
p_{12}^{\prime}
\end{array}\right]=\left[\begin{array}{cccccc}
c_{11} & c_{12} & c_{13} & 0 & 0 & 0 \\
c_{12} & c_{22} & c_{23} & 0 & 0 & 0 \\
c_{13} & c_{23} & c_{33} & 0 & 0 & 0 \\
0 & 0 & 0 & c_{44} & 0 & 0 \\
0 & 0 & 0 & 0 & c_{55} & 0 \\
0 & 0 & 0 & 0 & 0 & c_{66}
\end{array}\right]\left[\begin{array}{c}
e_{11}^{\prime} \\
e_{22}^{\prime} \\
e_{33}^{\prime} \\
2 e_{23}^{\prime} \\
2 e_{31}^{\prime} \\
2 e_{12}^{\prime}
\end{array}\right] .
$$

We consider a two-dimensional approximation in which the displacement components $\left(u_{1}, u_{2}, u_{3}\right)$ are independent of $x_{1}$ so that $\partial / \partial x_{1} \equiv 0$. Under this assumption the plane-strain problem $\left(u_{1}=0\right)$ and the antiplane strain problem $\left(u_{2}=u_{3}=0\right)$ are decoupled and, therefore, can be solved separately. The plane strain problem for an isotropic medium can be solved in terms of the Airy stress function $U$ such that

$$
\begin{aligned}
& p_{22}=\frac{\partial^{2} U}{\partial x_{3}^{2}}, \quad p_{33}=\frac{\partial^{2} U}{\partial x_{2}^{2}}, \quad p_{23}=-\frac{\partial^{2} U}{\partial x_{2} \partial x_{3}}, \\
& \nabla^{2} \nabla^{2} U=0 .
\end{aligned}
$$

The plane strain problem for an orthotropic medium can be solved in terms of the Airy stress function $U^{*}$ such that Garg et al (1991)

$$
\begin{aligned}
& p_{22}^{\prime}=\frac{\partial^{2} U^{*}}{\partial x_{3}^{2}}, \quad p_{33}^{\prime}=\frac{\partial^{2} U^{*}}{\partial x_{2}^{2}}, \quad p_{23}^{\prime}=-\frac{\partial^{2} U^{*}}{\partial x_{2} \partial x_{3}}, \\
& \left(a^{2} \frac{\partial^{2}}{\partial x_{2}^{2}}+\frac{\partial^{2}}{\partial x_{3}^{2}}\right)\left(b^{2} \frac{\partial^{2}}{\partial x_{2}^{2}}+\frac{\partial^{2}}{\partial x_{3}^{2}}\right) U^{*}=0,
\end{aligned}
$$


where

$$
a^{2}+b^{2}=\frac{\left(c_{22} c_{33}-c_{23}^{2}-2 c_{23} c_{44}\right)}{c_{33} c_{44}}, \quad a^{2} b^{2}=\frac{c_{22}}{c_{33}} .
$$

For an isotropic medium

$$
\begin{aligned}
& c_{11}=c_{22}=c_{33}=\frac{2 \mu(1-\sigma)}{1-2 \sigma}, \\
& c_{12}=c_{13}=c_{23}=\frac{2 \mu \sigma}{1-2 \sigma}, \\
& c_{44}=c_{55}=c_{66}=\mu .
\end{aligned}
$$

This yields $a^{2}=b^{2}=1$ and equation (6) reduces to equation (4).

As given by Singh \& Rani (1991), we have the Airy stress function due to a dip-slip line source parallel to the $x_{1}$-axis and passing through the point $\left(y_{2}, y_{3}\right)$ located in the isotropic half-space welded with the orthotropic half-space. For the isotropic half-space

$$
\begin{aligned}
U= & \frac{\alpha \mu \bar{b} d s}{\pi}\left[\operatorname { c o s } 2 \delta \left\{\frac{\left(x_{2}-y_{2}\right)\left(x_{3}-y_{3}\right)}{R^{2}}-2(D-C) \tan ^{-1}\left(\frac{x_{2}-y_{2}}{x_{3}+y_{3}}\right)\right.\right. \\
& \left.-\frac{\left(X_{1} y_{3}+X_{3} x_{3}\right)\left(x_{2}-y_{2}\right)}{S^{2}}-\frac{4 X_{2}\left(x_{2}-y_{2}\right) y_{3} x_{3}\left(x_{3}+y_{3}\right)}{S^{4}}\right\} \\
& +\sin 2 \delta\left\{-2(D-C) \ln S+\frac{\left(x_{3}-y_{3}\right)^{2}}{R^{2}}\right. \\
& \left.\left.+\frac{\left(X_{1} y_{3}+X_{3} x_{3}\right)\left(x_{3}+y_{3}\right)-2 X_{2} x_{3} y_{3}}{S^{2}}+\frac{4 X_{2} x_{3} y_{3}\left(x_{3}+y_{3}\right)^{2}}{S^{4}}\right\}\right],
\end{aligned}
$$

and for the orthotropic half-space

$$
\begin{aligned}
U^{*}= & \frac{2 \alpha \mu \bar{b} d s}{\pi}\left[\operatorname { c o s } 2 \delta \left\{C \tan ^{-1}\left(\frac{x_{2}-y_{2}}{y_{3}-a x_{3}}\right)\right.\right. \\
& \left.-D \tan ^{-1}\left(\frac{x_{2}-y_{2}}{y_{3}-b x_{3}}\right)-\left(x_{2}-y_{2}\right) y_{3}\left(\frac{A}{T^{2}}+\frac{B}{H^{2}}\right)\right\} \\
& \left.+\sin 2 \delta\left\{C \ln T-D \ln H+\frac{A y_{3}\left(y_{3}-a x_{3}\right)}{T^{2}}+\frac{B y_{3}\left(y_{3}-b x_{3}\right)}{H^{2}}\right\}\right],
\end{aligned}
$$

where

$$
\begin{aligned}
& \alpha=\frac{1}{2(1-\sigma)} \\
& \bar{b}=\text { displacement discontinuity } \\
& d s=\text { width of the line source } \\
& \delta=\text { dip angle }
\end{aligned}
$$




$$
\begin{aligned}
& \left(x_{2}, x_{3}\right)=\text { receiver location, } \\
& R^{2}=\left(x_{2}-y_{2}\right)^{2}+\left(x_{3}-y_{3}\right)^{2}, \quad S^{2}=\left(x_{2}-y_{2}\right)^{2}+\left(x_{3}+y_{3}\right)^{2}, \\
& T^{2}=\left(x_{2}-y_{2}\right)^{2}+\left(y_{3}-a x_{3}\right)^{2}, \quad H^{2}=\left(x_{2}-y_{2}\right)^{2}+\left(y_{3}-b x_{3}\right)^{2}, \\
& X_{1}=2(A+B)-1, \quad X_{2}=A(1+a)+B(1+b)-1, \\
& X_{3}=2 D(1+b)-2 C(1+a)+1, \quad A=\frac{\alpha\left(1-b+2 \mu s_{2}-2 \mu r_{2}\right)}{W}, \quad \quad \quad \quad r_{1}=\frac{\left(1+b-b \alpha+2 \mu \alpha s_{2}\right)}{W}, \\
& B=\frac{\alpha\left(a-1+2 \mu r_{1}-2 \mu s_{1}\right)}{W}, \quad s_{1}=\frac{c_{23} a^{2}+c_{22}}{a \Delta}, \\
& D=\frac{\left(1+a-a \alpha+2 \mu \alpha s_{1}\right)}{W}, \quad \Delta=\left(c_{22} c_{33}-c_{23}^{2}\right), \\
& r_{2}=\frac{c_{33} b^{2}+c_{23}}{\Delta}, \quad \\
& s_{2}=\frac{c_{23} b^{2}+c_{22}}{b \Delta}, \quad\left(1+a-\alpha+2 \mu \alpha r_{1}\right)\left(1+b-b \alpha+2 \mu \alpha s_{2}\right) \\
& W= \\
& \quad-\left(1+a-a \alpha+2 \mu \alpha s_{1}\right)\left(1+b-\alpha+2 \mu \alpha r_{2}\right) .
\end{aligned}
$$

Following Rani \& Bala (2006), using the polar co-ordinates (s, $\delta$ ) (figure 1a)

$$
y_{2}=s \cos \delta, y_{3}=d+s \sin \delta
$$

and integrating over s between the limits $(0, L)$, we obtain the following expressions for the Airy stress function for a long reverse dip-slip fault of width $L$ and infinite length with lower edge of the fault at distance $d$ from the interface:

$$
\begin{aligned}
U= & \frac{\alpha \mu \bar{b}}{\pi}\left[\left\{4(D-C)+\left(X_{1}+1\right)\right\} s \sin \delta \cos \delta\right. \\
& +\left\{2(D-C)\left(x_{2} \sin \delta-X^{\prime} \cos \delta-s \sin 2 \delta\right)+\left(X_{1} d+X_{3} x_{3}\right) \cos \delta\right. \\
& \left.+X_{1} x_{2} \sin \delta\right\} \ln S+\left(x_{2} \sin \delta-X \cos \delta\right) \ln R \\
& -2(D-C) s \cos 2 \delta \quad \tan ^{-1}\left(\frac{x_{2}-s \cos \delta}{X^{\prime}+s \sin \delta}\right) \\
& -2 X_{2} x_{3}\left(x_{2} \sin \delta+X^{\prime} \cos \delta\right)(d+s \sin \delta) \frac{1}{S^{2}} \\
& +\left\{x_{3} \sin \delta\left(-X_{1}+2 X_{2}+X_{3}\right)\right. \\
& \left.\left.-2(D-C)\left(x_{2} \cos \delta+X^{\prime} \sin \delta\right)\right\} \tan ^{-1}\left(\frac{s-x_{2} \cos \delta+X^{\prime} \sin \delta}{x_{2} \sin \delta+X^{\prime} \cos \delta}\right)\right]\left.\right|_{0} ^{L}
\end{aligned}
$$




$$
\begin{aligned}
U^{*}= & \frac{2 \alpha \mu \bar{b}}{\pi}[\{2(D-C)+(A+B)\} s \sin \delta \cos \delta \\
& +\left\{A\left(x_{2} \sin \delta+d \cos \delta\right)-C\left(x_{2} \sin \delta-Y \cos \delta-s \sin 2 \delta\right)\right\} \ln T \\
& +\left\{B\left(x_{2} \sin \delta+d \cos \delta\right)+D\left(x_{2} \sin \delta-Y^{\prime} \cos \delta-s \sin 2 \delta\right)\right\} \ln H \\
& +C s \cos 2 \delta \tan ^{-1}\left(\frac{x_{2}-s \cos \delta}{Y+s \sin \delta}\right)-D s \cos 2 \delta \tan ^{-1}\left(\frac{x_{2}-s \cos \delta}{Y^{\prime}+s \sin \delta}\right) \\
& +\left\{C\left(x_{2} \cos \delta+Y \sin \delta\right)+A a x_{3} \sin \delta\right\} \tan ^{-1}\left(\frac{s-x_{2} \cos \delta+Y \sin \delta}{x_{2} \sin \delta+Y \cos \delta}\right) \\
& \left.-\left\{D\left(x_{2} \cos \delta+Y^{\prime} \sin \delta\right)-B b x_{3} \sin \delta\right\} \tan ^{-1}\left(\frac{s-x_{2} \cos \delta+Y^{\prime} \sin \delta}{x_{2} \sin \delta+Y^{\prime} \cos \delta}\right)\right]\left.\right|_{0} ^{L},
\end{aligned}
$$
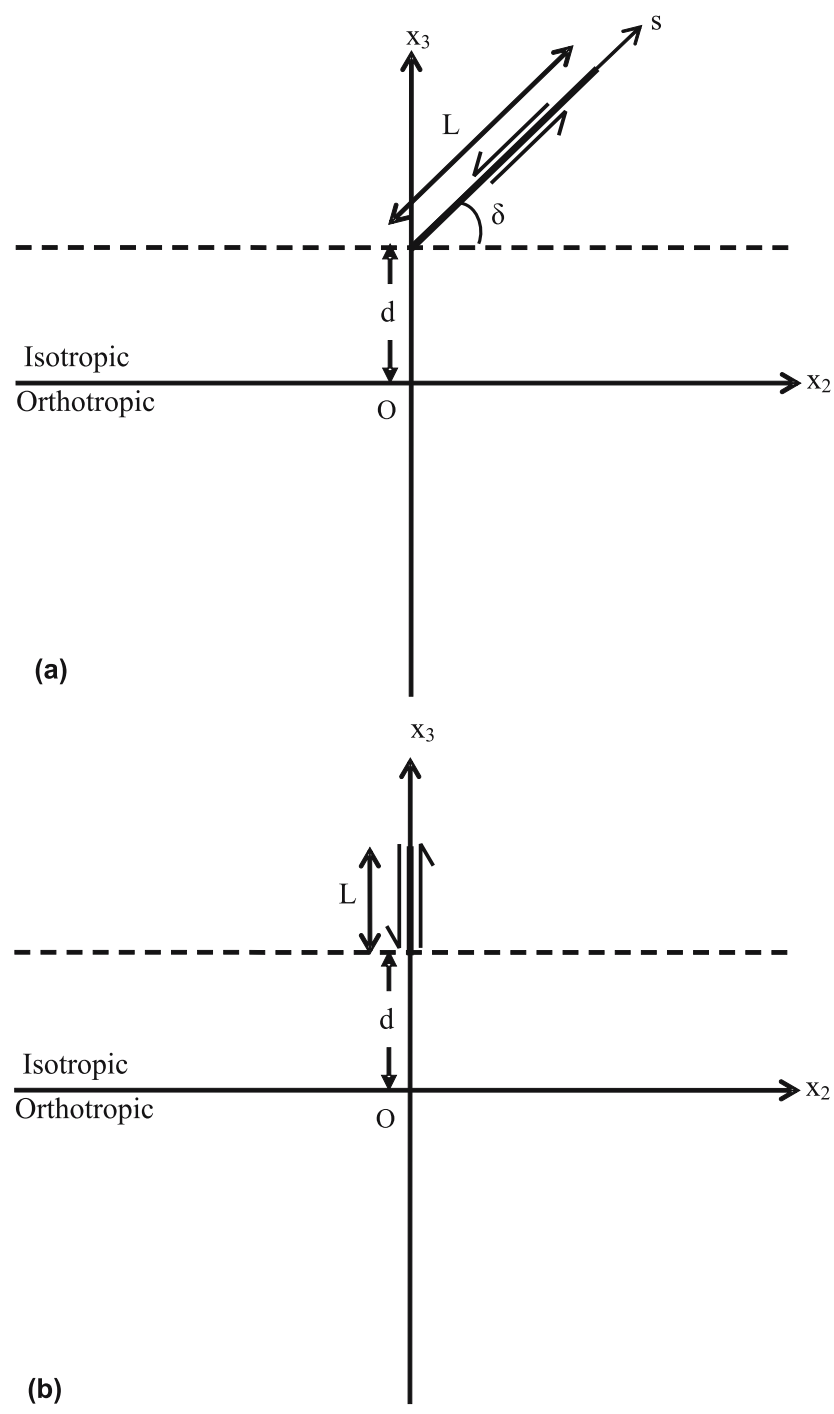

Figure 1. Geometry of a reverse dip-slip fault of width $L$ having lower edge at a distance $d$ from the interface in the isotropic halfspace in welded contact with an orthotropic half-space. (a) $\delta$ is the dip angle and $s$ is the distance from the lower edge of the fault measured in the dip direction, (b) $\delta=90^{\circ}$. 
where now

$$
\begin{aligned}
& R^{2}=\left(x_{2}-s \cos \delta\right)^{2}+(X-s \sin \delta)^{2}, \quad S^{2}=\left(x_{2}-s \cos \delta\right)^{2}+\left(X^{\prime}+s \sin \delta\right)^{2}, \\
& T^{2}=\left(x_{2}-s \cos \delta\right)^{2}+(Y+s \sin \delta)^{2}, \quad H^{2}=\left(x_{2}-s \cos \delta\right)^{2}+\left(Y^{\prime}+s \sin \delta\right)^{2}, \\
& X=x_{3}-d, \quad X^{\prime}=x_{3}+d, \quad Y=d-a x_{3}, Y^{\prime}=d-b x_{3}, \\
& \left.f(s)\right|_{0} ^{L}=f(L)-f(0) .
\end{aligned}
$$

\section{Stresses and displacements}

Using equations (3) and (12), we obtain the following expressions for the stresses of the isotropic medium:

$$
\begin{aligned}
& p_{22}=\frac{\alpha \mu \bar{b}}{\pi}\left[\left(x_{2} \sin \delta-3 X \cos \delta+s \sin 2 \delta\right) \frac{1}{R^{2}}\right. \\
& -2\left(x_{2} \sin \delta-X \cos \delta\right)(X-s \sin \delta)^{2} \frac{1}{R^{4}} \\
& +\left\{2\left(X_{3}-D+C\right)\left(x_{2} \sin \delta+X^{\prime} \cos \delta\right)\right. \\
& \left.+\left(4 X_{2}-X_{1}\right)\left(x_{2} \sin \delta-d \cos \delta-s \sin 2 \delta\right)+X_{3} x_{3} \cos \delta\right\} \frac{1}{S^{2}} \\
& -2\left\{X_{1}\left(x_{2} \sin \delta+X^{\prime} \cos \delta\right)\left(X^{\prime}+s \sin \delta\right)^{2}+2 X_{2} x_{3}^{2} \sin \delta\left(x_{2}-s \cos \delta\right)\right. \\
& -\left(X_{1}-X_{3}\right) x_{3}\left(X^{\prime}+s \sin \delta\right)\left(x_{2} \sin \delta+X^{\prime} \cos \delta\right) \\
& \left.-2 X_{2}(d+s \sin \delta)\left(X^{\prime}+s \sin \delta\right)\left[2\left(x_{2} \sin \delta+X^{\prime} \cos \delta\right)+3 x_{3} \cos \delta\right]\right\} \frac{1}{S^{4}} \\
& \left.-16 X_{2} x_{3}\left(x_{2} \sin \delta+X^{\prime} \cos \delta\right)(d+s \sin \delta)\left(X^{\prime}+s \sin \delta\right)^{2} \frac{1}{S^{6}}\right]\left.\right|_{0} ^{L}, \\
& p_{23}=\frac{\alpha \mu \bar{b}}{\pi}\left[\left(x_{2} \cos \delta+X \sin \delta-s\right) \frac{1}{R^{2}}\right. \\
& +2(X-s \sin \delta)^{2}\left(s-x_{2} \cos \delta-X \sin \delta\right) \frac{1}{R^{4}} \\
& +\left\{2(D-C)\left(x_{2} \cos \delta-X^{\prime} \sin \delta-s\right)-X_{1} x_{3} \sin \delta+4 X_{2} x_{3} \sin \delta\right. \\
& \left.+X_{3}\left(s+2 x_{3} \sin \delta+d \sin \delta-x_{2} \cos \delta\right)\right\} \frac{1}{S^{2}} \\
& +\left\{2 x_{3}\left(X_{1}-2 X_{2}\right)\left(X^{\prime}+s \sin \delta\right)^{2} \sin \delta\right.
\end{aligned}
$$




$$
\begin{aligned}
& -2 X_{1}\left(X^{\prime}+s \sin \delta\right)\left[X^{\prime} \sin \delta\left(X^{\prime}+s \sin \delta\right)-d \cos \delta\left(x_{2}-s \cos \delta\right)\right. \\
& \left.+s \sin \delta\left(s-x_{2} \cos \delta+X^{\prime} \sin \delta\right)\right]+4 X_{2}(d+s \sin \delta) \\
& \times\left[\left(x_{3}+X^{\prime}+s \sin \delta\right)\left(s-x_{2} \cos \delta+X^{\prime} \sin \delta\right)+2 x_{3} \sin \delta\left(X^{\prime}+s \sin \delta\right)\right] \\
& \left.+2 X_{3} x_{3}\left(X^{\prime}+s \sin \delta\right)\left(x_{2} \cos \delta-X^{\prime} \sin \delta-s\right)\right\} \frac{1}{S^{4}} \\
& \left.+16 X_{2} x_{3}(d+s \sin \delta)\left(X^{\prime}+s \sin \delta\right)^{2}\left(x_{2} \cos \delta-X^{\prime} \sin \delta-s\right) \frac{1}{S^{6}}\right]\left.\right|_{0} ^{L},
\end{aligned}
$$

$$
\begin{aligned}
p_{33}= & \frac{\alpha \mu \bar{b}}{\pi}\left[\left(x_{2} \sin \delta+X \cos \delta-s \sin 2 \delta\right) \frac{1}{R^{2}}\right. \\
& +2\left(x_{2} \sin \delta-X \cos \delta\right)(X-s \sin \delta)^{2} \frac{1}{R^{4}} \\
& +\left\{2(D-C)\left(x_{2} \sin \delta+X^{\prime} \cos \delta\right)\right. \\
& \left.+X_{1}\left(x_{2} \sin \delta-d \cos \delta-s \sin 2 \delta\right)-X_{3} x_{3} \cos \delta\right\} \frac{1}{S^{2}} \\
& +\left\{2 X _ { 1 } ( X ^ { \prime } + s \operatorname { s i n } \delta ) \left[\left(X^{\prime}+s \sin \delta\right)\left(x_{2} \sin \delta+d \cos \delta\right)\right.\right. \\
& \left.-x_{3} \sin \delta\left(x_{2}-s \cos \delta\right)\right]+4 X_{2} x_{3}\left[x_{3} \sin \delta\left(x_{2}-s \cos \delta\right)\right. \\
& \left.-3 \cos \delta\left(X^{\prime}+s \sin \delta\right)(d+s \sin \delta)\right] \\
& \left.+2 X_{3} x_{3}\left(X^{\prime}+s \sin \delta\right)\left(x_{2} \sin \delta+X^{\prime} \cos \delta\right)\right\} \frac{1}{S^{4}} \\
& \left.+16 X_{2} x_{3}\left(x_{2} \sin \delta+X^{\prime} \cos \delta\right)(d+s \sin \delta)\left(X^{\prime}+s \sin \delta\right)^{2} \frac{1}{S^{6}}\right]\left.\right|_{0} ^{L}
\end{aligned}
$$

The stresses for the orthotropic medium are obtained from equations (5) and (13). These are:

$$
\begin{aligned}
p_{22}^{\prime}= & \frac{2 \alpha \mu \bar{b}}{\pi}\left[a^{2}\left\{A\left(s \sin 2 \delta-x_{2} \sin \delta+d \cos \delta\right)+C\left(x_{2} \sin \delta+Y \cos \delta\right)\right\} \frac{1}{T^{2}}\right. \\
& +b^{2}\left\{B\left(s \sin 2 \delta-x_{2} \sin \delta+d \cos \delta\right)-D\left(x_{2} \sin \delta+Y^{\prime} \cos \delta\right)\right\} \frac{1}{H^{2}} \\
& -2 a^{2} A(d+s \sin \delta)\left(x_{2} \sin \delta+Y \cos \delta\right)(Y+s \sin \delta) \frac{1}{T^{4}} \\
& \left.-2 b^{2} B(d+s \sin \delta)\left(x_{2} \sin \delta+Y^{\prime} \cos \delta\right)\left(Y^{\prime}+s \sin \delta\right) \frac{1}{H^{4}}\right]\left.\right|_{0} ^{L}
\end{aligned}
$$




$$
\begin{aligned}
p_{23}^{\prime}= & \frac{2 \alpha \mu \bar{b}}{\pi}\left[a\left\{C\left(x_{2} \cos \delta-Y \sin \delta-s\right)-a A x_{3} \sin \delta\right\} \frac{1}{T^{2}}\right. \\
& -b\left\{D\left(x_{2} \cos \delta-Y^{\prime} \sin \delta-s\right)+b B x_{3} \sin \delta\right\} \frac{1}{H^{2}} \\
& -2 a A(d+s \sin \delta)(Y+s \sin \delta)\left(x_{2} \cos \delta-Y \sin \delta-s\right) \frac{1}{T^{4}} \\
& \left.-2 b B(d+s \sin \delta)\left(Y^{\prime}+s \sin \delta\right)\left(x_{2} \cos \delta-Y^{\prime} \sin \delta-s\right) \frac{1}{H^{4}}\right]\left.\right|_{0} ^{L} \\
p_{33}^{\prime}= & \frac{2 \alpha \mu \bar{b}}{\pi}\left[\left\{A\left(x_{2} \sin \delta-d \cos \delta-s \sin 2 \delta\right)-C\left(x_{2} \sin \delta+Y \cos \delta\right)\right\} \frac{1}{T^{2}}\right. \\
& +\left\{B\left(x_{2} \sin \delta-d \cos \delta-s \sin 2 \delta\right)+D\left(x_{2} \sin \delta+Y^{\prime} \cos \delta\right)\right\} \frac{1}{H^{2}} \\
& +2 A(d+s \sin \delta)(Y+s \sin \delta)\left(x_{2} \sin \delta+Y \cos \delta\right) \frac{1}{T^{4}} \\
& \left.+2 B(d+s \sin \delta)\left(Y^{\prime}+s \sin \delta\right)\left(x_{2} \sin \delta+Y^{\prime} \cos \delta\right) \frac{1}{H^{4}}\right]\left.\right|_{0} ^{L}
\end{aligned}
$$

The displacements, for the isotropic half-space, are given by the relations (Singh \& Rani 1991)

$$
\begin{aligned}
& 2 \mu u_{2}=-\frac{\partial U}{\partial x_{2}}+\frac{1}{2 \alpha} \int\left(p_{22}+p_{33}\right) d x_{2}, \\
& 2 \mu u_{3}=-\frac{\partial U}{\partial x_{3}}+\frac{1}{2 \alpha} \int\left(p_{22}+p_{33}\right) d x_{3} .
\end{aligned}
$$

The displacements, for the orthotropic half-space, are given by the expressions (Singh \& Rani 1991)

$$
\begin{aligned}
& u_{2}^{\prime}=\frac{1}{\Delta} \int\left(c_{33} p_{22}^{\prime}-c_{23} p_{33}^{\prime}\right) d x_{2}, \\
& u_{3}^{\prime}=\frac{1}{\Delta} \int\left(c_{22} p_{33}^{\prime}-c_{23} p_{22}^{\prime}\right) d x_{3},
\end{aligned}
$$

where

$$
\Delta=c_{22} c_{33}-c_{23}^{2} \text {. }
$$

Corresponding to the stresses (15)-(17), the displacements for the isotropic half-space are

$$
\begin{aligned}
u_{2}= & \frac{\alpha \bar{b}}{2 \pi}\left[-\left(1-\frac{1}{\alpha}\right) \sin \delta \ln R-\left\{2(D-C)+X_{1}-\frac{\left(X_{3}+2 X_{2}\right)}{\alpha}\right\} \sin \delta \ln S\right. \\
& -\frac{\cos \delta}{\alpha}\left\{\tan ^{-1}\left(\frac{x_{2}-s \cos \delta}{X-s \sin \delta}\right)-X_{3} \tan ^{-1}\left(\frac{x_{2}-s \cos \delta}{X^{\prime}+s \sin \delta}\right)\right\} \\
& +2(D-C) \cos \delta \tan ^{-1}\left(\frac{s-x_{2} \cos \delta+X^{\prime} \sin \delta}{x_{2} \sin \delta+X^{\prime} \cos \delta}\right)
\end{aligned}
$$




$$
\begin{aligned}
+ & (X-s \sin \delta)\left(x_{2} \cos \delta+X \sin \delta-s\right) \frac{1}{R^{2}} \\
+ & \left\{\left(X_{1}-\frac{2 X_{2}}{\alpha}\right)(d+s \sin \delta)\left(s-x_{2} \cos \delta+X^{\prime} \sin \delta\right)\right. \\
+ & \left.x_{3}\left[2 X_{2} x_{3} \sin \delta+X_{3}\left(s-x_{2} \cos \delta+X^{\prime} \sin \delta\right)\right]\right\} \frac{1}{S^{2}} \\
+ & \left.4 X_{2} x_{3}\left(s-x_{2} \cos \delta+X^{\prime} \sin \delta\right)(d+s \sin \delta)\left(X^{\prime}+s \sin \delta\right) \frac{1}{S^{4}}\right]\left.\right|_{0} ^{L}, \\
u_{3}= & \frac{\alpha \bar{b}}{2 \pi}\left[\left(1-\frac{1}{\alpha}\right) \cos \delta \ln R+\left\{2(D-C)-X_{3}\left(1-\frac{1}{\alpha}\right)\right\} \cos \delta \ln S\right. \\
+ & \frac{\sin \delta}{\alpha}\left\{\tan -1\left(\frac{X-s \sin \delta}{x_{2}-s \cos \delta}\right)+\left(2 X_{2}+X_{3}\right) \tan -1\left(\frac{X^{\prime}+s \sin \delta}{x_{2}-s \cos \delta}\right)\right\} \\
+ & \left\{2(D-C)+X_{1}-2 X_{2}-X_{3}\right\} \sin \delta \tan { }^{-1}\left(\frac{s-x_{2} \cos \delta+X^{\prime} \sin \delta}{x_{2} \sin \delta+X^{\prime} \cos \delta}\right) \\
& -\left(x_{2} \sin \delta-X \cos \delta\right)(X-s \sin \delta) \frac{1}{R^{2}} \\
+ & \left\{\left(-X_{1}+2 X_{2}-\frac{2 X_{2}}{\alpha}\right)(d+s \sin \delta)\left(x_{2} \sin \delta+X^{\prime} \cos \delta\right)\right. \\
+ & \left.2 X_{2} x_{3}\left(s \sin 2 \delta-x_{2} \sin \delta+d \cos \delta\right)-X_{3} x_{3}\left(x_{2} \sin \delta+X^{\prime} \cos \delta\right)\right\} \frac{1}{S^{2}} \\
& \left.-4 X_{2} x_{3}(d+s \sin \delta)\left(x_{2} \sin \delta+X^{\prime} \cos \delta\right)\left(X^{\prime}+s \sin \delta\right) \frac{1}{S^{4}}\right]\left.\right|_{0} ^{L}
\end{aligned}
$$

Corresponding to the stresses (18)-(20), the displacements for the orthotropic half-space are

$$
\begin{aligned}
u_{2}^{\prime}= & -\frac{2 \alpha \mu \bar{b}}{\pi}\left[(A-C) r_{1} \sin \delta \ln T+(B+D) r_{2} \sin \delta \ln H\right. \\
& +A r_{1}(d+s \sin \delta)\left(x_{2} \cos \delta-Y \sin \delta-s\right) \frac{1}{T^{2}} \\
& +B r_{2}(d+s \sin \delta)\left(x_{2} \cos \delta-Y^{\prime} \sin \delta-s\right) \frac{1}{H^{2}} \\
& \left.-C r_{1} \cos \delta \tan ^{-1}\left(\frac{x_{2}-s \cos \delta}{Y+s \sin \delta}\right)+D r_{2} \cos \delta \tan ^{-1}\left(\frac{x_{2}-s \cos \delta}{Y^{\prime}+s \sin \delta}\right)\right]\left.\right|_{0} ^{L} \\
u_{3}^{\prime}= & \frac{2 \alpha \mu \bar{b}}{\pi}\left[C s_{1} \cos \delta \ln T-D s_{2} \cos \delta \ln H\right. \\
& +A s_{1}(d+s \sin \delta)\left(x_{2} \sin \delta+Y \cos \delta\right) \frac{1}{T^{2}} \\
& +B s_{2}(d+s \sin \delta)\left(x_{2} \sin \delta+Y^{\prime} \cos \delta\right) \frac{1}{H^{2}}
\end{aligned}
$$




$$
\begin{aligned}
& -(A-C) s_{1} \sin \delta \tan ^{-1}\left(\frac{Y+s \sin \delta}{x_{2}-s \cos \delta}\right) \\
& \left.-(B+D) s_{2} \sin \delta \tan ^{-1}\left(\frac{Y^{\prime}+s \sin \delta}{x_{2}-s \cos \delta}\right)\right]\left.\right|_{0} ^{L} .
\end{aligned}
$$

The stresses given by (15)-(20) and the displacements given by (24)-(27) are the results for a long dip-slip fault of finite width $L$ located in the isotropic half-space overlying the orthotropic half-space. The results obtained here satisfy the necessary continuity conditions

$$
\begin{gathered}
p_{23}=p_{23}^{\prime}, \quad p_{33}=p_{33}^{\prime}, \\
u_{2}=u_{2}^{\prime}, \quad u_{3}=u_{3}^{\prime} .
\end{gathered}
$$

At $z=0$ for the two half-spaces to be in welded contact along the plane $z=0$. Moreover, when the orthotropic half-space is replaced by the isotropic one, the results of the present paper, in the limit, coincide with the corresponding results of Rani \& Bala (2006) for two isotropic half-spaces in welded contact.

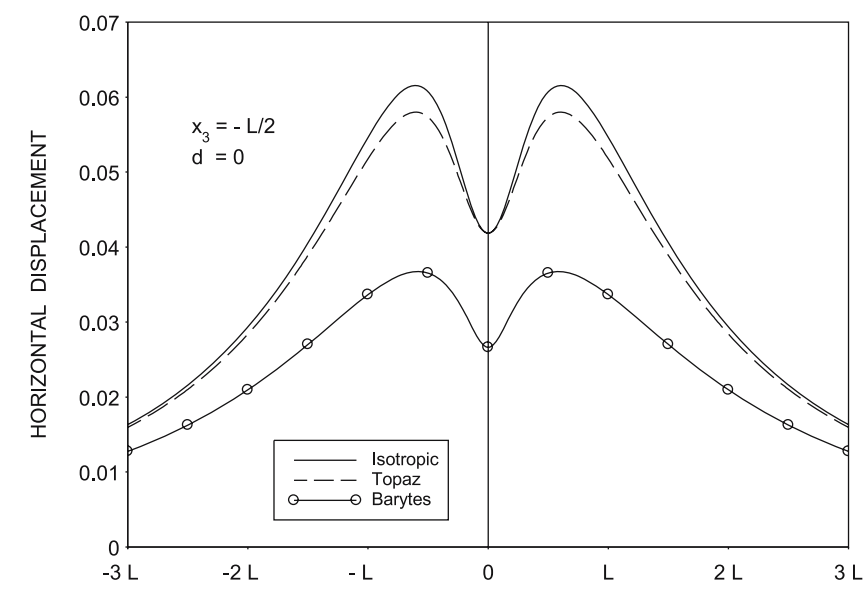

(a)

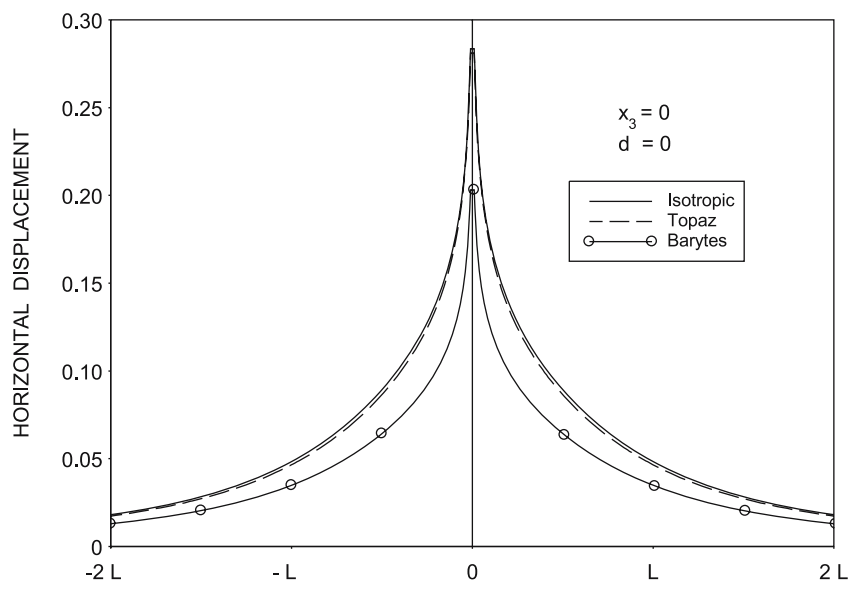

(b)
Figure 2. Variation of the horizontal displacement $\left(u_{2}^{\prime} / \bar{b}\right)$ with the distance from the fault due to interface-breaking vertical dipslip fault $(d=0)$ for (a) $x_{3}=$ $-L / 2$, (b) $x_{3}=0$. 


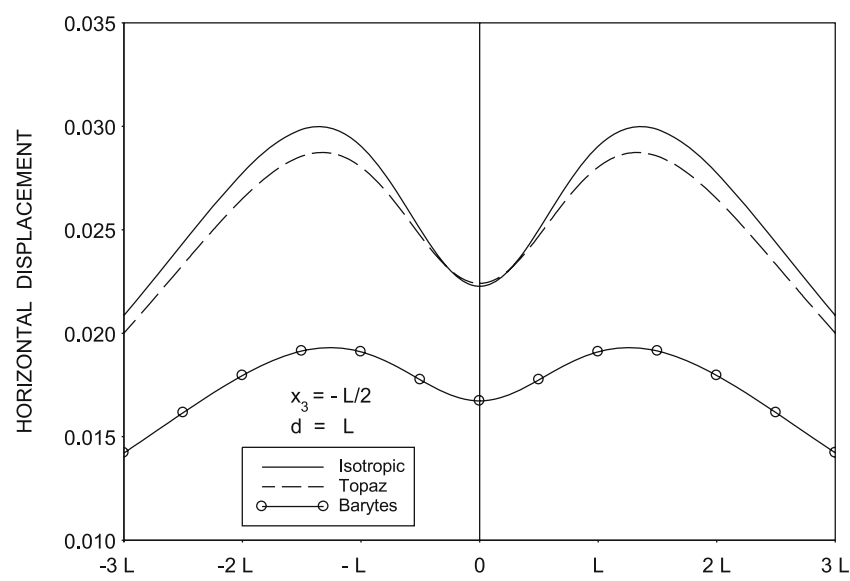

(a) DISTANCE FROM THE FAULT

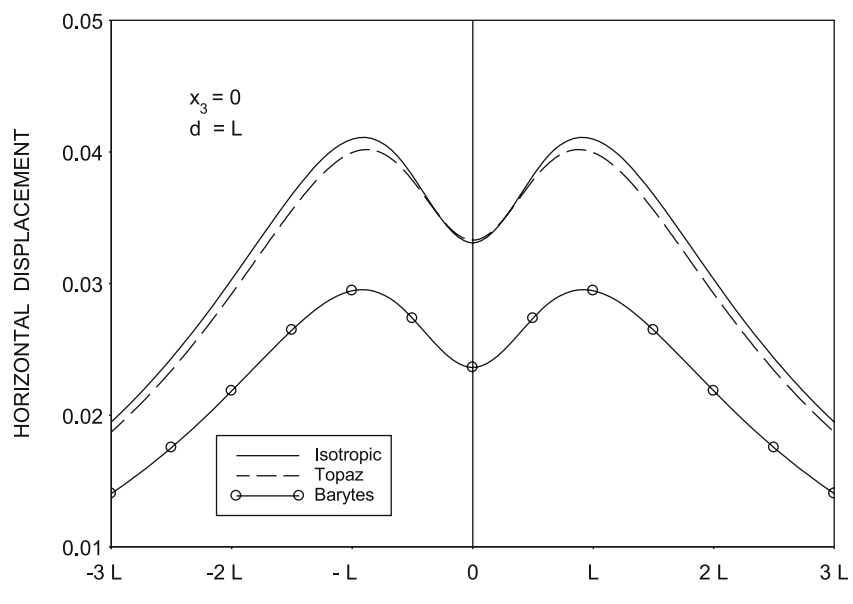

(b)

DISTANCE FROM THE FAULT

Figure 3. Variation of the horizontal displacement $\left(u_{2}^{\prime} / \bar{b}\right)$ with the distance from the fault due to a long vertical dip-slip fault at distance $L$ from the interface for (a) $x_{3}=-L / 2$, (b) $x_{3}=0$.

\section{Numerical results}

We compare the displacement field due to a long vertical dip-slip fault of width $L$ with its edge at the distance $d$ from the interface located in the isotropic half-space welded with orthotropic half-space along the horizontal plane with the corresponding displacement field when both the half-spaces are isotropic. Therefore, we take $\delta=90^{\circ}$ (figure 1b). We assume the isotropic half-space to be Poissonian so that $\sigma=0 \cdot 25$. For the orthotropic half-space, we use the values of elastic constants given by Love (1944). For Topaz,

$$
\begin{aligned}
& c_{11}=2870, c_{22}=3560, c_{33}=3000, \\
& c_{12}=1280, c_{23}=900, c_{13}=860, \\
& c_{44}=1100, c_{55}=1350, c_{66}=1330,
\end{aligned}
$$

in terms of a unit stress of $10^{6}$ grammes wt $/ \mathrm{cm}^{2}$, this yields $a=1.2992$ and $b=0.8385$. 


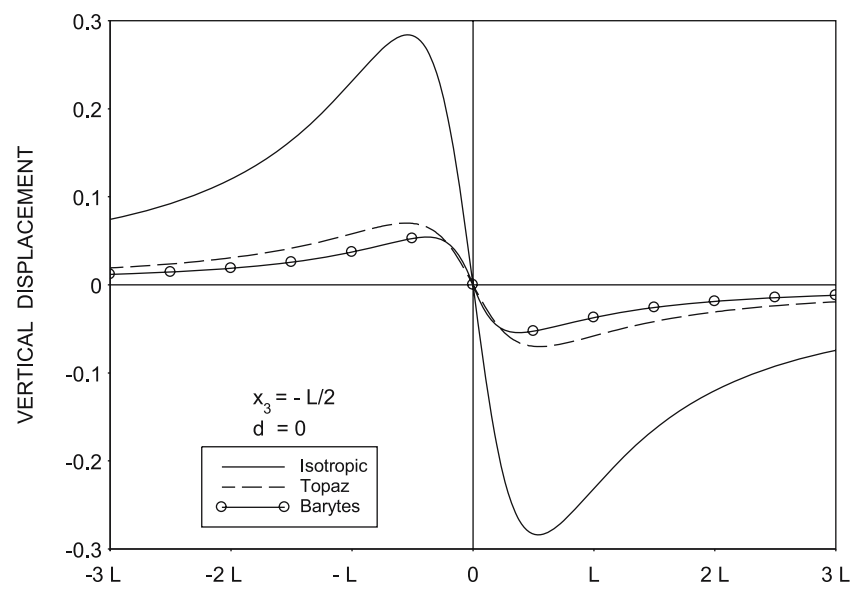

(a) DISTANCE FROM THE FAULT

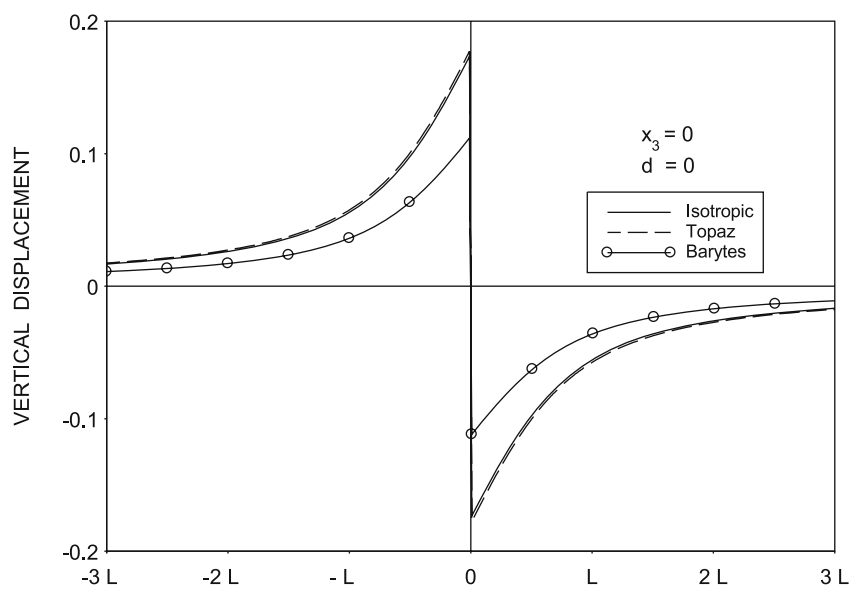

(b)

DISTANCE FROM THE FAULT

Figure 4. Variation of vertical displacement $\left(u_{3}^{\prime} / \bar{b}\right)$ with the distance from the fault due to interface breaking dip-slip fault $(d=0)$ for (a) $x_{3}=-L / 2$, (b) $x_{3}=0$.

For Barytes,

$$
\begin{aligned}
& c_{11}=907, c_{22}=800, c_{33}=1074, \\
& c_{12}=468, c_{23}=273, c_{13}=275, \\
& c_{44}=122, c_{55}=293, c_{66}=283,
\end{aligned}
$$

in terms of $10^{6}$ grammes $\mathrm{wt} / \mathrm{cm}^{2}$, we obtain $a=2 \cdot 3118$ and $b=0.3735$.

When the lower half-space is also isotropic,

$$
\begin{aligned}
& c_{11}=c_{22}=c_{33}=\frac{2 \mu^{\prime}\left(1-\sigma^{\prime}\right)}{1-2 \sigma^{\prime}}, \\
& c_{12}=c_{13}=c_{23}=\frac{2 \mu^{\prime} \sigma^{\prime}}{1-2 \sigma^{\prime}}, \\
& c_{44}=c_{55}=c_{66}=\mu^{\prime} .
\end{aligned}
$$

We take $\sigma^{\prime}=0.25$ and $c_{44} / \mu=0.5$ for numerical computations. 


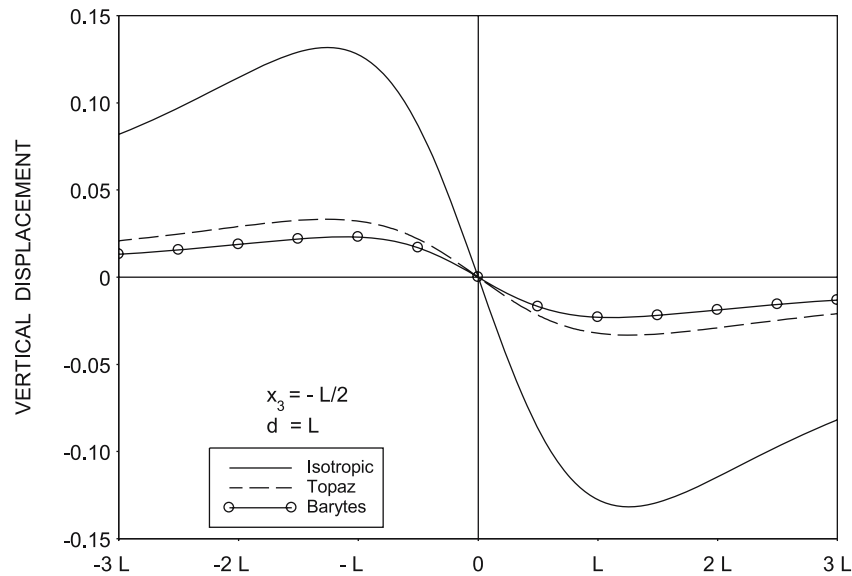

(a)

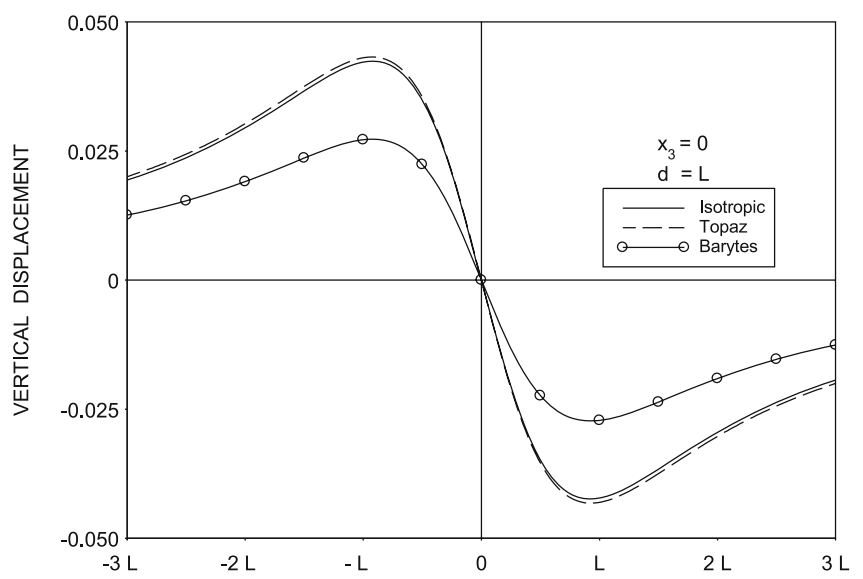

(b)

DISTANCE FROM THE FAULT
Figure 5. Variation of vertical displacement $\left(u_{3}^{\prime} / \bar{b}\right)$ with the distance from the fault due to a long vertical dip-slip fault at distance $L$ from the interface for (a) $x_{3}=$ $-L / 2$, (b) $x_{3}=0$.

Figures $2 \mathrm{a}-\mathrm{b}$ display the horizontal displacement $\left(u_{2}^{\prime} / \bar{b}\right)$ with the distance from the fault due to interface breaking fault $(d=0)$. The horizontal displacement is symmetric about the line $x_{2}=0$. In figure $2 \mathrm{a}$, the observer is in the orthotropic half-space at $x_{3}=-L / 2$. The horizontal displacement for Barytes varies more significantly in magnitude rather than Topaz from the corresponding one for the isotropic case. In figure $2 b$, the observer is at the interface, the horizontal displacement attains maximum value at origin. Figures $3 \mathrm{a}-\mathrm{b}$ show the horizontal displacement $\left(u_{2}^{\prime} / \bar{b}\right)$ with the distance from the fault located at distance $L$ from the interface. In figure $3 \mathrm{a}$, the observer is in the orthotropic half-space at $x_{3}=-L / 2$ but in figure $3 b$, the observer is at the interface. We observe that magnitude of the displacement for Barytes is affected significantly, but the pattern is not affected much.

Figures $4 \mathrm{a}-\mathrm{b}$ exhibit the vertical displacement $\left(u_{3}^{\prime} / \bar{b}\right)$ with the distance from the fault due to interface breaking fault $(d=0)$. The displacement is antisymmetric about the line $x_{2}=0$. The effect of anisotropy on the magnitude of the displacement is more pronounced when the observer is in orthotropic half-space. When the observer is at the interface $x_{3}=$ 0 , the displacement is singular at the lower edge point. Figures $5 \mathrm{a}-\mathrm{b}$ display the vertical 


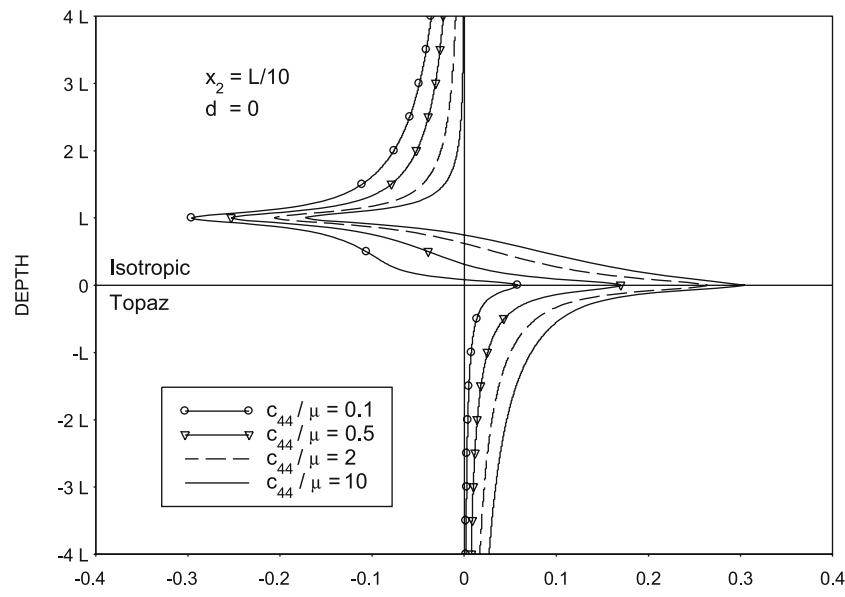

(a)

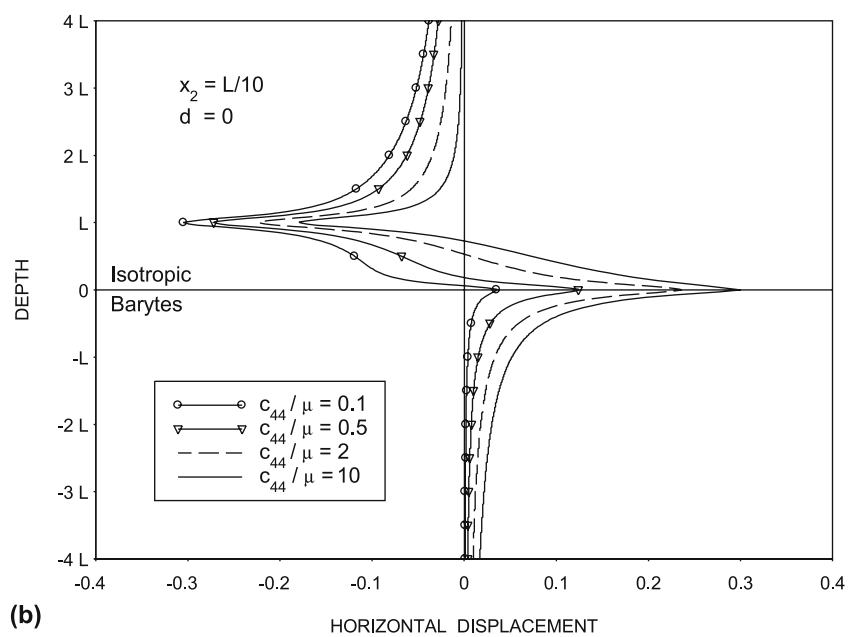

Figure 6. Variation of the horizontal displacement $\left(u_{2}^{\prime} / \bar{b}\right)$ with depth at $x_{2}=L / 10$ for interface breaking fault for different values of ratio of rigidities $c_{44} / \mu=0 \cdot 1,0 \cdot 5,2$ and 10 taking the orthotropic material as (a) Topaz (b) Barytes. The magnitude of the displacement is affected.

displacement $\left(u_{3}^{\prime} / \bar{b}\right)$ for a buried fault at a distance $L$ from the interface. We note that there is a strong variation in the vertical displacement for Barytes as well as Topaz from the vertical displacement for the isotropic case when the observer is in the orthotropic half-space.

Next, we compute the effect of ratio of rigidities of the two media on the displacement field for two different orthotropic materials, namely Topaz and Barytes. Figures $6 \mathrm{a}-\mathrm{b}$ show the variation of the horizontal displacement in unit of $\bar{b}$ with depth for four values of $c_{44} / \mu=$ $0 \cdot 1,0 \cdot 5,2$ and 10 for Topaz and Barytes, respectively, for interface breaking fault. For all values of ratio of rigidities, the horizontal displacement attains maximum value at the lower end of the fault and minimum value at the upper end of the fault. Figures $7 \mathrm{a}-\mathrm{b}$ are for the vertical displacement. The values of the ratio of rigidities affect the magnitude of the horizontal and the vertical displacement in a reverse way. The horizontal displacement varies more significantly in the orthotropic half-space as well as in the isotropic half-space. But the vertical displacement varies in the orthotropic half-space and up to fault depth in the isotropic half-space significantly. 


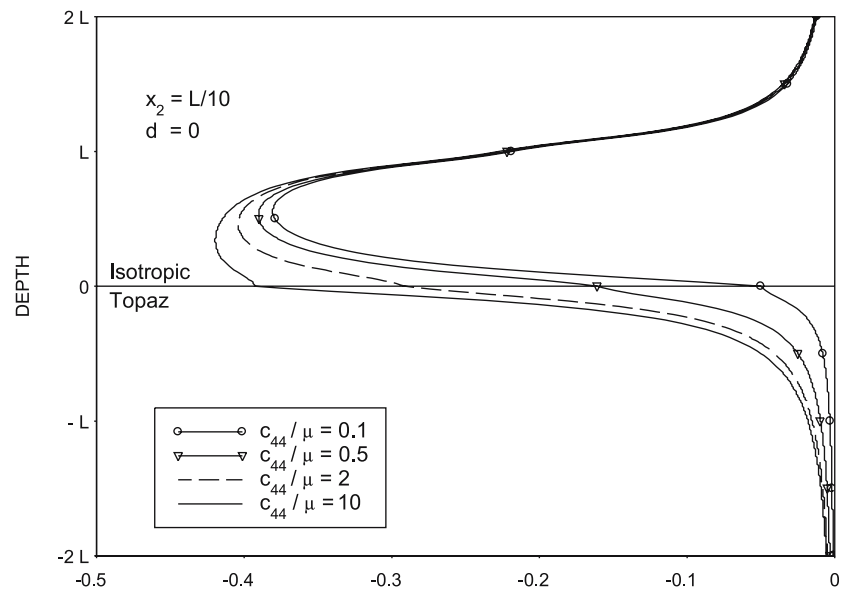

(a)

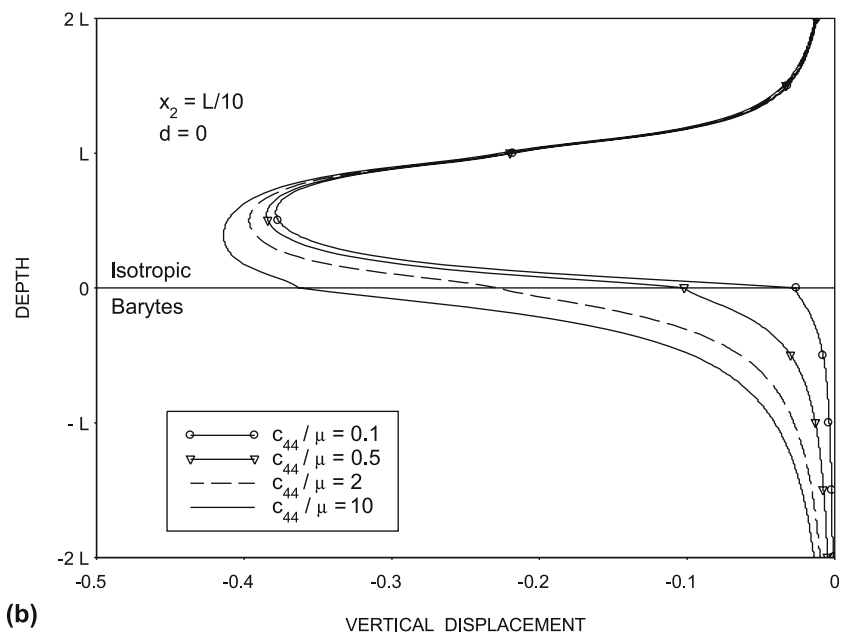

Figure 7. Variation of the vertical displacement $\left(u_{3}^{\prime} / \bar{b}\right)$ with depth at $x_{2}=L / 10$ for interface breaking fault for different values of ratio of rigidities $c_{44} / \mu=0 \cdot 1,0 \cdot 5,2$ and 10 taking the orthotropic material as (a) Topaz (b) Barytes.

\section{Conclusions}

We have obtained the closed-form expressions for the elastic residual field caused by a long dip-slip fault of finite width located in an isotropic half-space overlying an orthotropic halfspace. The displacement field caused by a long dip-slip fault of width $L$ has been compared with the displacement field when both the half-spaces are isotropic. Two orthotropic materials namely Topaz and Barytes have been considered for numerical computations. Numerical results show that the effect of anisotropy on the displacement field is more pronounced when the observer is in the orthotropic half-space. In case of horizontal displacement, there is a strong variation in the behaviour of Barytes from the corresponding isotropic case, but the vertical displacement for Barytes as well as for Topaz varies significantly from the corresponding isotropic case. The rock forming minerals of deep crust and upper mantle of the earth exhibit orthotropic symmetry, the results obtained may find applications in geophysical engineering. 


\section{References}

Ben-Zion Y 1989 The response of two joined quarter spaces to SH line sources located at material discontinuity interface. Geophy. J. Int. 98: 213-222

Bonafede M, Rivalta L 1999 On tensile cracks close to and across the interface between two welded elastic half-spaces. Geophys. J. Int. 138: 410-434

Garg N R, Madan D K, Sharma R K 1996 Two-dimensional deformation of an orthotropic elastic medium due to seismic sources. Phys. Earth Planet. Inter. 94: 43-62

Garg N R, Singh S J, Manchanda S 1991 Static deformation of an orthotropic multilayered elastic halfspace by two-dimensional surface loads. Proc. Ind. Acad. Sci. (Earth Planet. Sci.) 100: 205-218

Kumar A, Singh S J, Singh J 2002 Static deformation of two welded monoclinic elastic half-spaces due to a long inclined strike-slip fault. Proc. Indian Acad. Sci. (Earth Planet. Sci.) 111: 125-131

Kumar A, Singh S J, Singh J 2005 Deformation of two welded elastic half-spaces due to a long inclined tensile fault. J. Earth Syst. Sci. 114: 97-103

Rani S, Bala N 2006 2-D deformation of two welded half-spaces due to a blind dip-slip fault. J. Earth Syst. Sci. 115: 277-287

Rani S, Singh S J 1992 Static deformation of two welded half-spaces due to dip-slip faulting. Proc. Indian Acad. Sci. (Earth Planet. Sci.) 101: 269-282

Singh S J, Rani S 1991 Static deformation of two-dimensional seismic sources embedded in an isotropic half-space in welded contact with an orthotropic half-space. J. Phys. Earth. 39: 599-618

Singh S J, Rani S, Garg N R 1992 Displacements and stresses in two welded half-spaces due to twodimensional sources. Phys. Earth Planet. Int. 70: 90-101

Wu R S, Chou Y T 1982 Line force in a two-phase orthotropic medium, ASME J. Appl. Mech. 49: 55-61 